Original Research Paper

\title{
Graphene Synthesis by Plasma-Enhanced CVD Growth with Ethanol
}

\author{
${ }^{1}$ Teresa Campo, ${ }^{2}$ María Cotto, ${ }^{2}$ Francisco Márquez, ${ }^{1}$ Eduardo Elizalde and ${ }^{1}$ Carmen Morant \\ ${ }^{I}$ Department of Applied Physics, M-XII, Universidad Autónoma de Madrid, Cantoblanco, 28049 Madrid, Spain \\ ${ }^{2}$ Nanomaterials Research Group, School of Natural Sciences and Technology, University of Turabo, 00778PR, USA
}

Article history

Received: 17-06-2016

Revised: 21-06-2016

Accepted: 21-06-2016

Corresponding Author:

Teresa Campo

Department of Applied Physics,

M-XII, Universidad Autónoma

de Madrid, Cantoblanco, 28049

Madrid, Spain

Email: teresa.campo@uam.es

\begin{abstract}
A modified route to synthesize graphene flakes is proposed using the Chemical Vapor Deposition (CVD) technique, by using copper substrates as supports. The carbon source used was ethanol, the synthesis temperature was $950^{\circ} \mathrm{C}$ and the pressure was controlled along the whole process. In this CVD synthesis process the incorporation of the carbon source was produced at low pressure and $950^{\circ} \mathrm{C}$ inducing the appearance of a plasma blue flash inside the quartz tube. Apparently, the presence of this plasma blue flash is required for obtaining graphene flakes. The synthesized graphene was characterized by different techniques, showing the presence of non-oxidized graphene with high purity.
\end{abstract}

Keywords: Graphene Flakes, Chemical Vapor Deposition, PlasmaEnhanced, AFM, Raman Spectroscopy

\section{Introduction}

The science of carbon materials is in continuous progress, being one of the most active and multidisciplinary areas of Science. Among the carbon materials, graphene has become a rising star on the horizon of material science. Due to its unique planar structure, transparency, mechanical strength, thermal properties and electronic conductivity (Dacheng and Yunqi, 2010; Phaedon, 2010; Fisichella et al., 2013) graphene is a very promising material for the development of nanoelectronic devices, sensors, energy-storage and/or transparent conducting electrodes applications (Phaedon, 2010; Zhang et al., 2013; Schwierz, 2010; Guo et al., 2011). The exceptional properties of graphene are a consequence of the continuous network of hexagonally arranged $\mathrm{sp}^{2}$-bonded carbon atoms in a 2D-structure.

Among the different synthesis processes to obtain graphene (i.e., chemical exfoliation, mechanical cleavage, epitaxial growth or Chemical Vapor DepositionCVD), the last one (CVD) is considered as the most promising procedure to obtain continuous graphene flakes with low level of defects. Although the presence of unwanted by-products and structural damages is inevitable, this method is one of the most suitable for large-scale and controllable synthesis of graphene. Commonly, the synthesis of graphene by CVD requires a copper or nickel sheet as substrate (Zhang et al., 2013; Edwards and Coleman, 2013; Dacheng et al., 2013;
Batzill, 2012) and alcohols or methane as carbon source (Zhang et al., 2013; Guermoune et al., 2011; Campos-Delgado et al., 2013).

In this research, the CVD method previously described for the growth of carbon nanotubes (Morant et al., 2012) was slightly modified to obtain graphene. Thus, a mixture of ethanol: $\mathrm{N}_{2}: \mathrm{H}_{2}$ was used to obtain a plasma discharge at high temperature, responsible for the synthesis of graphene. A complete analysis of the as-synthesized graphene flakes has been performed using a combination of tools including Scanning and transmission Electron Microscopies (SEM and TEM), Raman spectroscopy, X-ray Photoemission Spectroscopy (XPS), Atomic Force Microscopy (AFM) and infrared spectroscopy (FT-IR).

\section{Experimental Details}

\section{Preparation of Substrates}

Polycrystalline copper samples (Goodfellow 99.9\%), with areas of $0.8 \times 1.2 \mathrm{~cm}\left(0.75 \mathrm{~cm}^{2}\right)$ and $300 \mu \mathrm{m}$ thickness, were used as substrates. As reported in the literature (Dacheng et al., 2013), copper samples were cleaned with acetic acid before growth. Different cleaning times of substrates with $1 \mathrm{M}$ acetic acid at $60^{\circ} \mathrm{C}$ and room temperature were tested. After cleaning, copper substrates were characterized by SEM, allowing to conclude that the best treatment was the use of $1 \mathrm{M}$ acetic 
acid aqueous solution for $2 \mathrm{~min}$ at room temperature. Subsequently, a cleaning procedure was carried out using acetone and isopropyl alcohol for $10 \mathrm{~min}$.

\section{Synthesis of Graphene}

After cleaning, copper substrates were placed into a quartz boat and introduced in a cylindrical quartz reactor (inner diameter of $25 \mathrm{~mm}$ and $1 \mathrm{~m}$-length) within a tube furnace. The quartz reactor was maintained at an initial vacuum of $10^{-2}$ Torr using a mechanical pump.

Figure 1 shows the scheme of the synthesis procedure. The temperature was risen to $950^{\circ} \mathrm{C}$, at a rate of $30^{\circ} \mathrm{C} / \mathrm{min}$ (Guermoune et al., 2011). Samples were maintained at this temperature for 26 min, using an $\mathrm{H}_{2}$ constant flow of $10 \mathrm{sccm}$ to remove possible organic impurities. After the first $20 \mathrm{~min}$ of this step, a flow mixture of $\mathrm{N}_{2}$ gas and ethanol vapor (30 $\left.\mathrm{sccm}\right)$, previously generated by an overpressure of $\mathrm{N}_{2}$, was introduced into the reactor. In this moment, a blue plasma discharge was generated. At the same time, the reactor pressure increases to $10^{2}$ Torr during a few seconds until the pressure stabilizes at 1 Torr. Once the growth time finishes, a cooling process takes place. During the first $30 \mathrm{~min}$ of the cooling step, a constant flow of $\mathrm{H}_{2} 10 \mathrm{sccm}$ is maintained and, after that, an $\mathrm{Ar}$ flow of $300 \mathrm{sccm}$ is introduced into the reactor.

\section{Graphene Transfer on Different Supports}

To analyze the graphene, synthesized on both faces of the copper substrates, it is required to transfer it to other different supports. The selected supports depend on the chosen analysis technique. For example, $\mathrm{SiO}_{2} / \mathrm{Si}$ support is appropriate for Raman microscopy and FTIR and copper patterned grids are the selected supports for subsequent characterization by TEM.

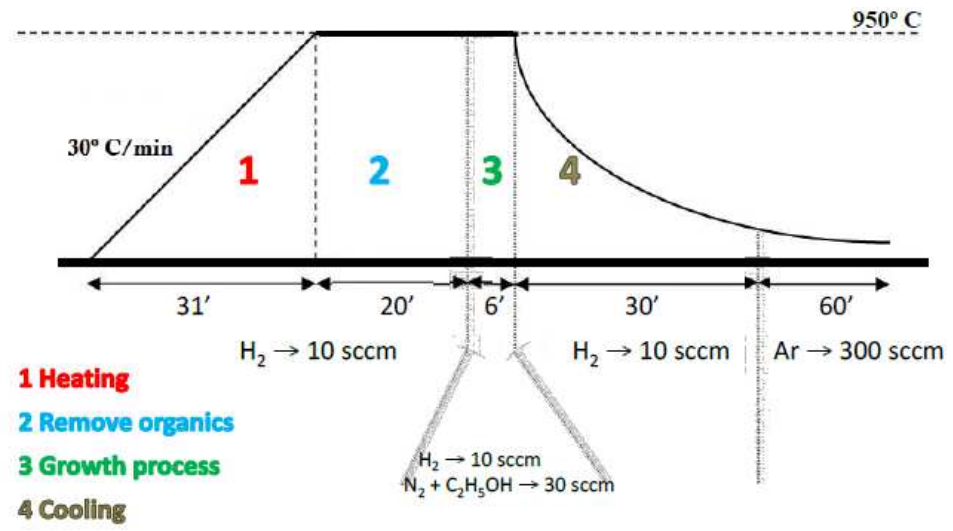

Fig. 1. Experimental conditions (temperature and reactive gases) used for the graphene synthesis

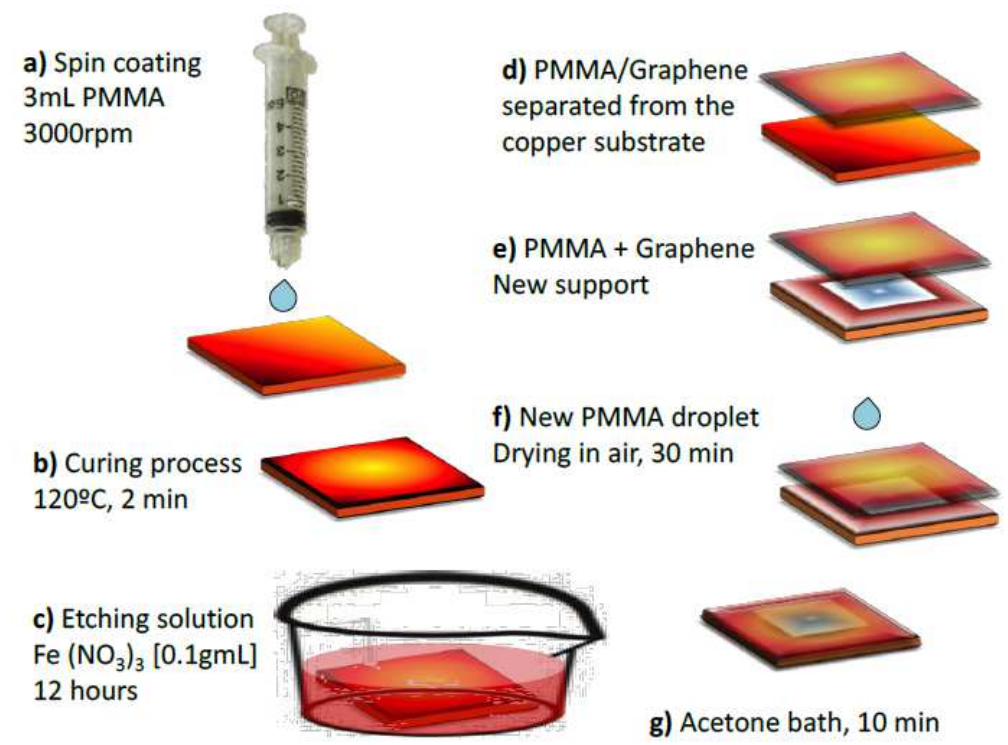

Fig. 2. Scheme of the transfer process of graphene from copper foil to a silicon substrate by using PMMA 
Figure 2 shows the different steps involved in the graphene transfer from copper foils. This procedure consists of several steps: (a) Deposition of $3 \mathrm{~mL}$ of Polymethyl methacrylate (PMMA) by spin-coating at $3000 \mathrm{rpm}$ on the copper substrate covered with graphene; (b) The curing process of PMMA is performed in an oven at $120^{\circ} \mathrm{C}$ for $2 \mathrm{~min}$; (c) the PMMA/sample is cooled down to room temperature and subsequently placed inside a $\mathrm{Fe}\left(\mathrm{NO}_{3}\right)_{3}\left[0.1 \mathrm{~g} \mathrm{~mL}^{-1}\right]$ etching solution for $12 \mathrm{~h}$; (d) after that period of time, the PMMA/graphene is separated from copper substrate and it can be clearly observed floating on the liquid surface; (e) PMMA/graphene assembly is cleaned with deionized water and deposited on the chosen support; (f) a small drop of PMMA is added on the assembly to eliminate the stress that may have occurred in the graphene during the transfer process; and (g) finally, this droplet of PMMA is dried for $30 \mathrm{~min}$ in air and the polymer is removed with acetone, leaving the graphene on the support and completing the transfer process.

\section{Characterization Techniques}

The morphology and structure of the synthesized graphene was studied by SEM (Philips, FEG XL-30S, at 20 $\mathrm{kV}$ ) and HRTEM (JEOL JEM $3000 \mathrm{~F}$, at $300 \mathrm{kV}$ ). The instrument used for Raman characterization was a Confocal 3D Raman Microscope Alpha 300 of WITec Focus Innovations. The selected laser excitation wavelength was $532 \mathrm{~nm}\left(\mathrm{Ar}^{+}\right)$, with $7.6 \mathrm{~mW}$ power. AFM images were collected operating in contact mode at room temperature and in ambient air conditions. The cantilevers used, with rectangular cross sections and normal force constant of 0.05 $\mathrm{N} / \mathrm{m}$, were made of $\mathrm{Si}_{3} \mathrm{~N}_{4}$ (Olympus NL levers). Finally, the FT-IR spectra were measured by using a FTIR Bruker IFS66v spectrometer, in specular reflectance mode, using an attenuated total reflection accessory.

\section{Results and Discussion}

\section{Characterization by Scanning and Transmission Electron Microscopies}

Figure 3 shows the SEM images of the copper substrate and graphene grown on copper substrate.
Figure $3 \mathrm{a}$ shows the copper substrate after a thermal treatment without carbon source. The copper grain boundaries can be observed, as well as slip planes and some erosion caused by the thermal treatment. Figure $3 \mathrm{~b}$ shows a thin grey film of the as-synthesized graphene covering the copper substrate. Here, the graphene shows wrinkles, observed in Fig. $3 \mathrm{~b}$ as white and parallel lines, that probably were generated by the difference in the coefficient of thermal expansion between graphene and copper (Zhang et al., 2013; Guermoune et al., 2011; Xuesong et al., 2009). The dark areas observed in Fig. $3 \mathrm{~b}$ correspond to the presence of thicker graphene multilayers. Figure $3 c$ shows an HRSEM image of a graphene monolayer on a copper hole produced by the previous chemical etching.

A comparative of the as-synthesized graphene and the subsequent transfer of graphene on a silicon wafer is shown in Fig. 4. Figure 4a shows that the graphene layers are coating the whole copper substrate. In the Raman optical image (Fig. 4b) the lightest zones correspond to silicon, while the darker to graphene. The incomplete coverage of the silicon wafer can be probably due to the difficulty of the PMMA transfer process.

Graphene was characterized by HRTEM. Graphene layers were supported on copper grids (Quantifoil 300 mesh), using the PMMA process described above. Figure 5a shows the SEM image of the graphene flakes deposited on a copper grid used for HRTEM. As can be seen there, graphene is found mainly in the upper left corner of the image. Figure 5b shows the HRTEM image of graphene. In spite of the strained process suffered by the graphene in the transfer by PMMA, we can observe some local ordered crystal structures with different orientations. The general evaluation, however, is that the observed graphene flakes are twisted, bended, wrinkled and partially folded on the grid. Isolated graphene flakes commonly behave in that way due to their high reactivity, resulting in their corrugation and scrolling (Meyer et al., 2007). Figure 5c presents a closer view of Fig. 5b, where the inset image corresponds to the FT of the marked region in the micrograph, which clearly shows the typical hexagonal crystal lattice (Guoxiu et al., 2008), being indicative of the presence of graphene flakes.

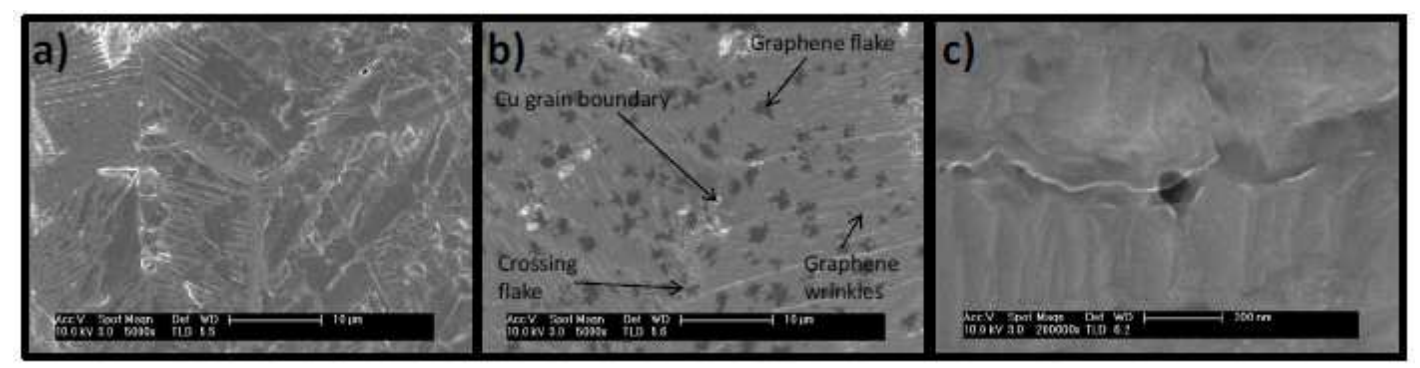

Fig. 3. Graphene SEM images of copper substrate after thermal treatment without carbon source (a); copper substrate covered by graphene (b); and a graphene monolayer on a copper hole 


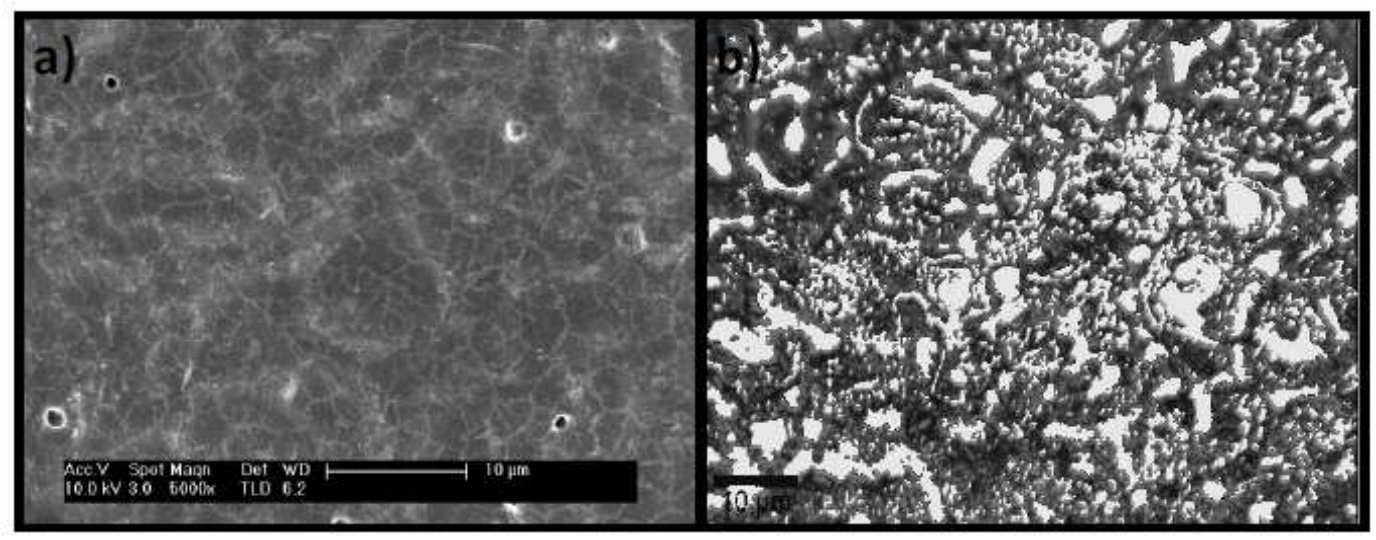

Fig. 4. SEM image of graphene/Cu (a); and Raman optical image of graphene/Si (b)

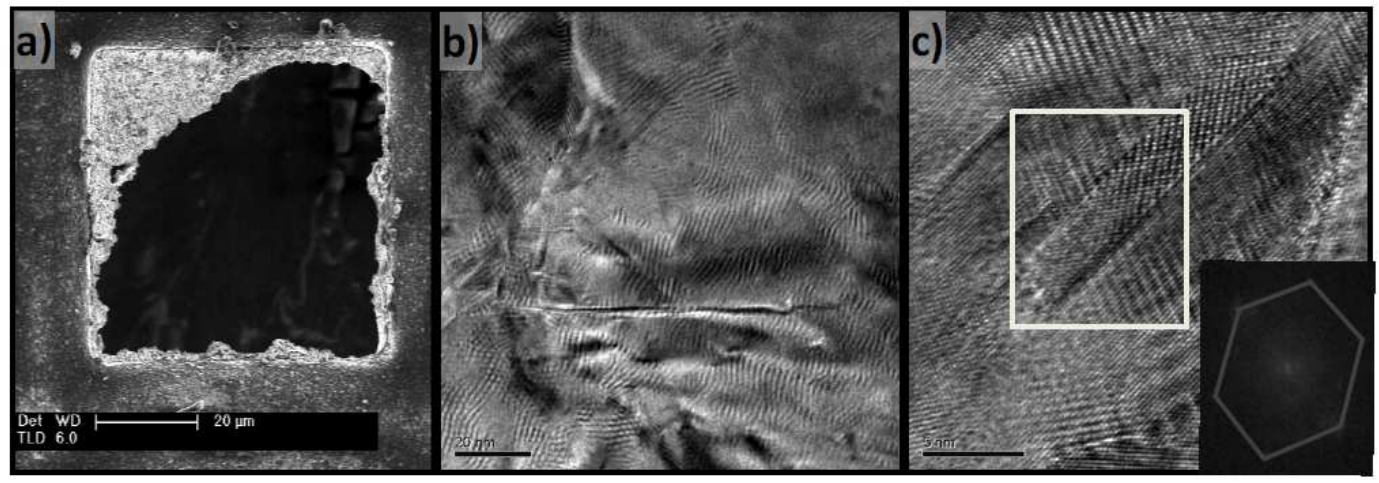

Fig. 5. SEM image of graphene supported on a copper grid (a); TEM Images of graphene flakes at different magnification (b) and (c). The inset corresponds to the FT pattern obtained from the marked region of image c
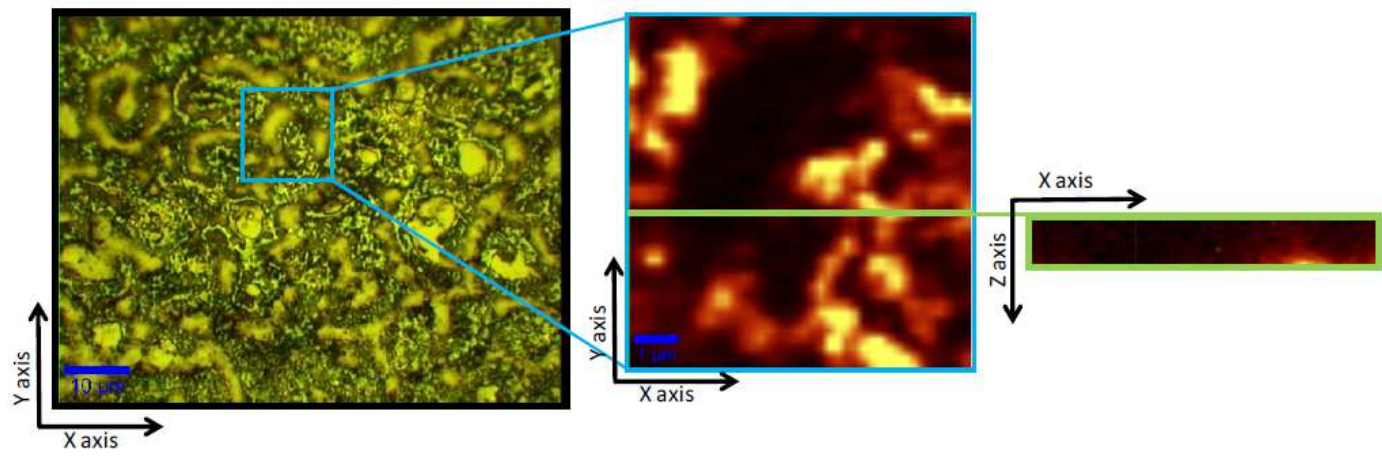

Fig. 6. Raman mapping spectra and linear spectra for the selected area and path, respectively

\section{Raman Characterization}

Raman spectroscopy is commonly used to study carbon materials (Kudin et al., 2008; Dresselhaus et al., 2010; Malard et al., 2009). The quality and uniformity of the grown graphene flakes, as well as the number of stacked layers and the presence of defects produced by chemical impurities and amorphous carbon, can be evaluated by Raman spectroscopy.
To avoid the copper signal from the substrates, the synthesized, graphene flakes were transferred by the PMMA process previously described and supported on silicon substrates. Samples were analyzed at room temperature and atmospheric pressure. The Confocal 3D Raman spectroscopy allows the acquisition of high resolution Raman spectra by exploring the $\mathrm{Z}$-axis corresponding to the height and depth of the sample (Fig. 6). 

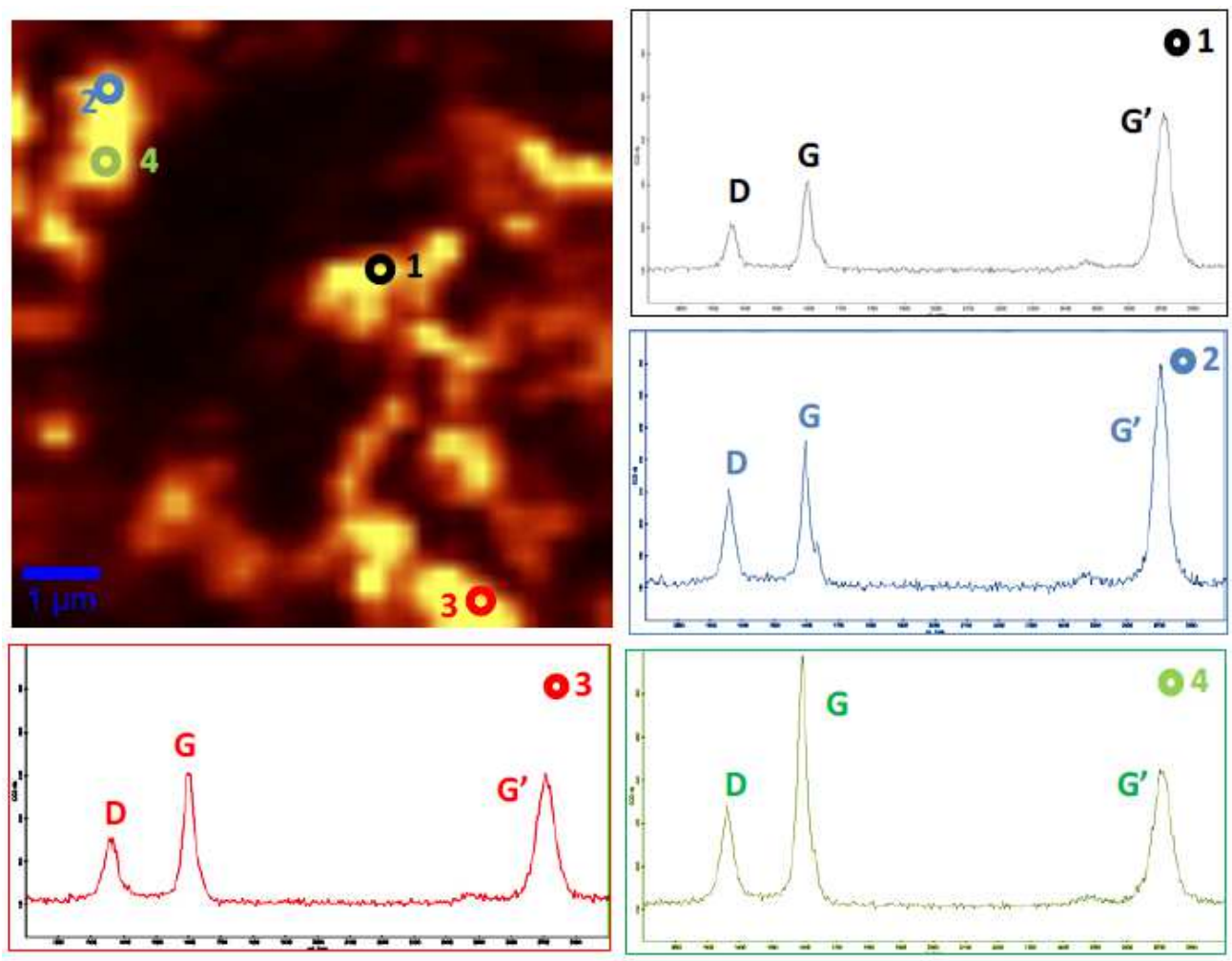

Fig. 7. 2D-Raman image of the synthesized graphene and the corresponding spectra in different points of the mapping

The Raman mapping spectra were indicative of the high purity of the graphene layers as well as the absence of defects. We have chosen four representative points (Fig. 7) to extract the Raman spectra and analyse them. The D, G and $\mathrm{G}^{\prime}$ bands are observed in all spectra with interesting differences in intensities and frequencies.

The existence of the D band in the range of 1362$1367 \mathrm{~cm}^{-1}$ indicates a certain density of defects present in the as-grown material. It can be explained by the PMMA transfer process of the graphene to the silicon substrate, which increases the defect amount of the graphene sample. The intensity of the $\mathrm{D}$ band in the spectra of Fig. 7 is relatively low, except on point 2. At this point the intensity of the $\mathrm{D}$ band increases, due to the fact that the measurement was done near graphene edges and domain boundaries.

The $\mathrm{G}$ band, which is common to all $\mathrm{sp}^{2}$ carbon forms, is present in the spectral range of 1596-1604 cm1. A widening of this band is indicative of an increased presence of defects in graphene. The $\mathrm{G}^{\prime}$ band appears in the spectral range of $2705-2715 \mathrm{~cm}^{-1}$. These bands $(\mathrm{G}$ and $\mathrm{G}^{\prime}$ ) are significant in providing information about the electronic and geometrical structure. From the intensity ratio $\mathrm{G} / \mathrm{G}^{\prime}$ it can be estimated the number of graphene stacked layers (Xuesong et al., 2009). If this ratio is approximately 1 it means that graphene was synthesized as a bilayer; if this ratio is $<1$ graphene is as a single layer; and finally if this ratio is $>1$ more than 2 layers of graphene (normally between 2 and 5) were obtained. According to our values, we can conclude that the number of graphene layers is less than 5 , because for more than 5 layers the Raman spectrum is practically equal to that of bulk graphite (Ferrari, 2007).

The single layer structure in points 1 and 2 is verified by the $\mathrm{G} / \mathrm{G}^{\prime}$ intensity ratio of 0.59 and 0.66 , respectively and a symmetric $G^{\prime}$ band with a full width at halfmaximum of $51-55 \mathrm{~cm}^{-1}$ (Gao et al., 2010). Point 3 has a much broader $G^{\prime}$ band with respect to graphene monolayer (points 1 and 2) which indicates the presence of graphene as a double layer, being this result corroborated by the $\mathrm{G} / \mathrm{G}^{\prime}$ intensity ratio of 1.01 (Table 1 ).

Quantifying disorder in graphene is usually made by analyzing the $\mathrm{D} / \mathrm{G}$ intensity ratio. As can be seen in Table 1 , the point 4 is characterized by having a lo level of defects while the point with more defects corresponds to point 2 . Points 1 and 3 have practically the same defect amount.

It is worth mentioning that after Raman analysis of all synthesized samples, we observed that when the blue plasma is not present (i.e., when the pressure of the ethanol- $\mathrm{N}_{2}$ mixture is not the appropriate one), graphene is not synthesized (Raman spectra not shown). So we can conclude that, in our experimental 
conditions, the blue plasma enhanced growth is required to obtain graphene flakes.

\section{XPS Characterization}

A detailed chemical analysis of the graphene grown on copper substrates using X-ray Photoelectron Spectroscopy (XPS) was performed. This technique is very useful to determine the chemical composition of the as-synthesized graphene. Although graphene was grown under vacuum and reductive conditions, a small percent of Graphene Oxide (GO) is expected, due to the oxygen rich carbon source (ethanol) and other oxygen sources. Therefore, for comparative purposes, beside the as-synthesized graphene, two other samples have been analyzed. One of them is a commercial GO sample (Varela-Rizo et al., 2010) and the other one is a GO sample after a reduced treatment (R-GO) by hydroiodic acid (Songfeng et al., 2010).

$\mathrm{Cu} 2 \mathrm{p}, \mathrm{O} 1 \mathrm{~s}$ and $\mathrm{C} 1 \mathrm{~s}$ regions have been recorded as shown in Fig. 4. The binding energy was calibrated using the $\mathrm{Cu} 2 \mathrm{p}$ core level peak, related to the copper oxidized form $\mathrm{CuO}$ at $933.6 \mathrm{eV}$. The spectra were normalized to the same intensity.

Figure $4 \mathrm{a}$ shows the $\mathrm{Cu} 2 \mathrm{p}$ spectrum of the as synthesized graphene. The $\mathrm{O} 1 \mathrm{~s}$ spectrum presents a narrow and sharped peak that can be assigned to the copper oxide (Fig. 4b). The areas of the O $1 \mathrm{~s}$ spectrum and those of the $\mathrm{Cu} 2 \mathrm{p}$ are in concordance with the oxidized form of copper $\mathrm{CuO}$. Therefore, there is no oxygen linked to graphene.

Figure $4 \mathrm{c}$ shows three $\mathrm{C}$ 1s spectra corresponding to GO, R-GO and as-synthesized graphene samples. The GO spectrum shows two components, one of them at $284.5 \mathrm{eV}$ that was assigned to $\mathrm{C}-\mathrm{C} \mathrm{sp} \mathrm{sp}^{2}$ bond. The other one is a shoulder in the region of $286-287 \mathrm{eV}$ that can be assigned to the C-O bond (Guermoune et al., 2011; Casero et al., 2013; Goldoni et al., 2002). On the contrary, the rest of the spectra corresponding to the
RGO and the as-synthesized graphene samples, show only the $\mathrm{C}-\mathrm{C} \mathrm{sp}^{2}$ peak. This fact unambiguously indicates that there is no oxygen presence in both samples. Then, we can conclude that although our synthesis method uses an oxygen rich carbon source, we obtained free-oxygen graphene samples.

\section{Atomic Force Microscopy Characterization}

As complementary characterization technique AFM was used to study the morphology and the comparative friction behavior between the clean copper substrate and the graphene grown on the copper surface.

Figure 8a shows the AFM image corresponding to a clean copper substrate after thermal treatment and in absence of any carbon source. Figure $8 \mathrm{~b}$ shows the AFM image of graphene growth on the same copper substrate observed in Fig. 8a.

According to these images, the morphology of both samples is clearly different. The copper substrate presents their grains delimitated by well-defined lines. On the other hand, the graphene on copper is smoothed and it is shown as a tissue over the copper structure.

During the graphene growth on copper, the different thermal expansion coefficients of graphene and $\mathrm{Cu}$ cause the appearance of multiple cracks due to the strain suffered by the materials (Fig. 9a). These surface features are completely different when the CVD process is performed without carbon source, as can be seen in Fig. 9b (Troppenz et al., 2013). The extra corrugation resulting from this strain relaxation is evaluated in Fig. 9c. The green and blue lines observed in Fig. 9a and 9b, respectively, show the place where the AFM line-scans were taken. The linescan rough mean squared roughness of the graphene grown on copper is $2.18 \mathrm{~nm}$, meanwhile on the copper clean substrate is $0.07 \mathrm{~nm}$, more than 30 times smaller.

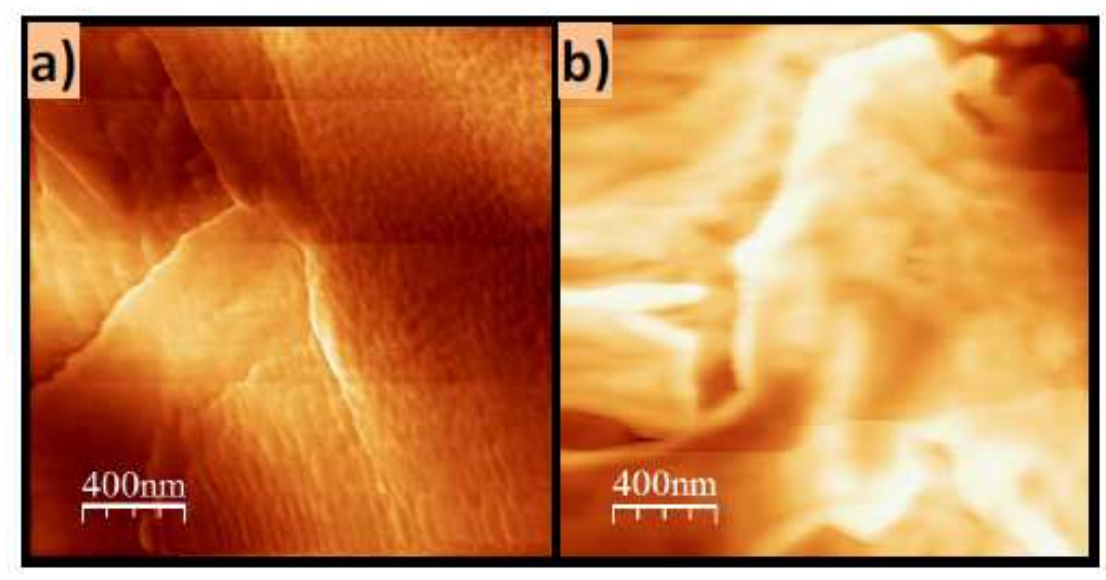

Fig. 8. AFM images corresponding to the same copper substrate before (a) and after graphene growth (b) 


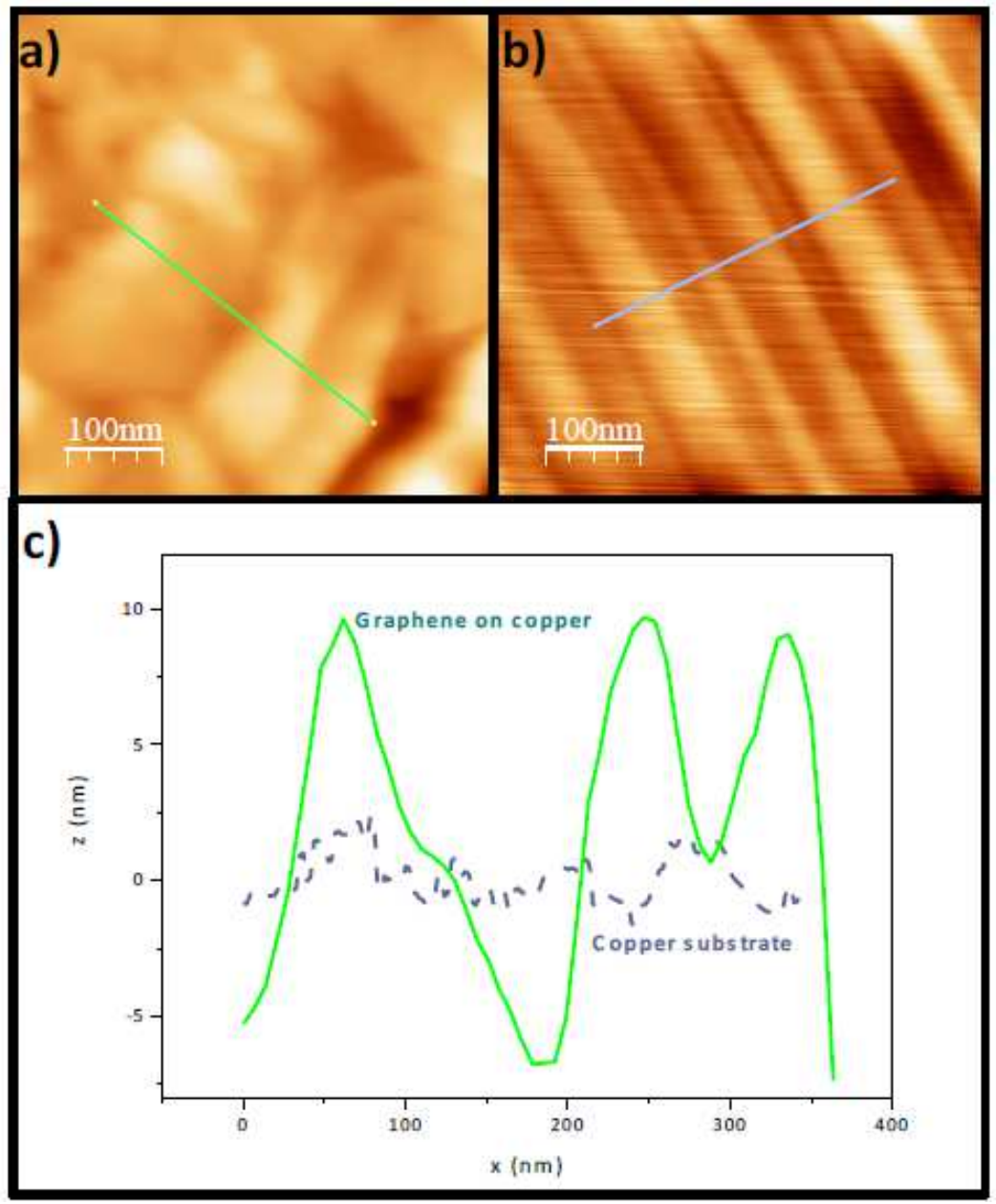

Fig. 9. AFM micrographs of graphene on Copper (a); Cu substrate (b); and comparative AFM line-scans (c)

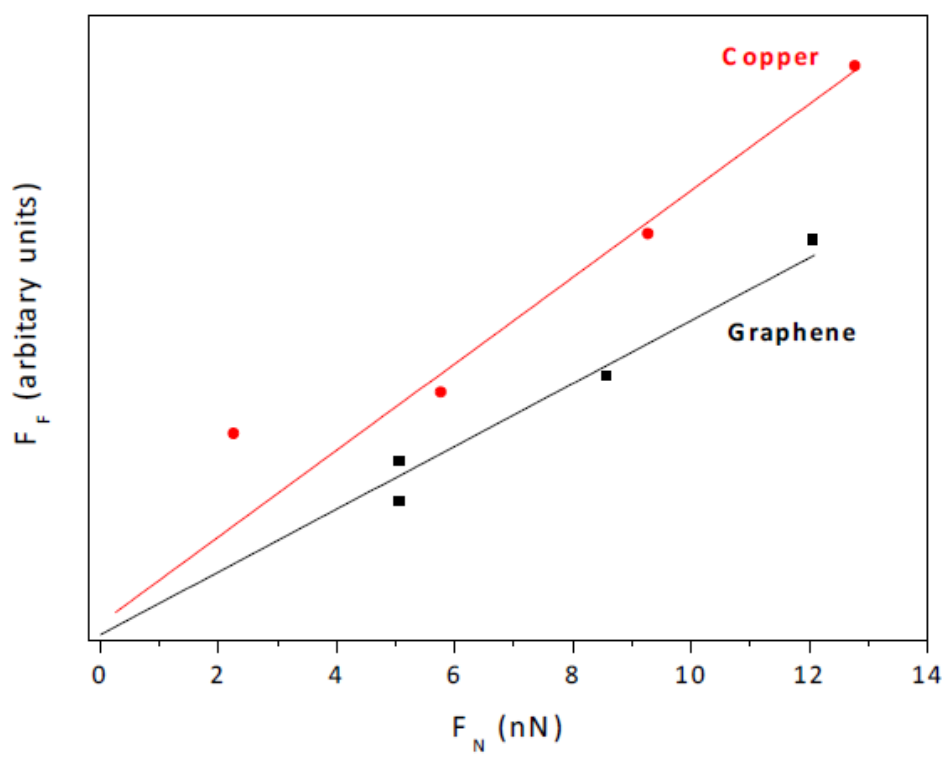

Fig. 10. Friction force Vs normal force measured by AFM for copper substrate (red circles) and graphene (black squares) 


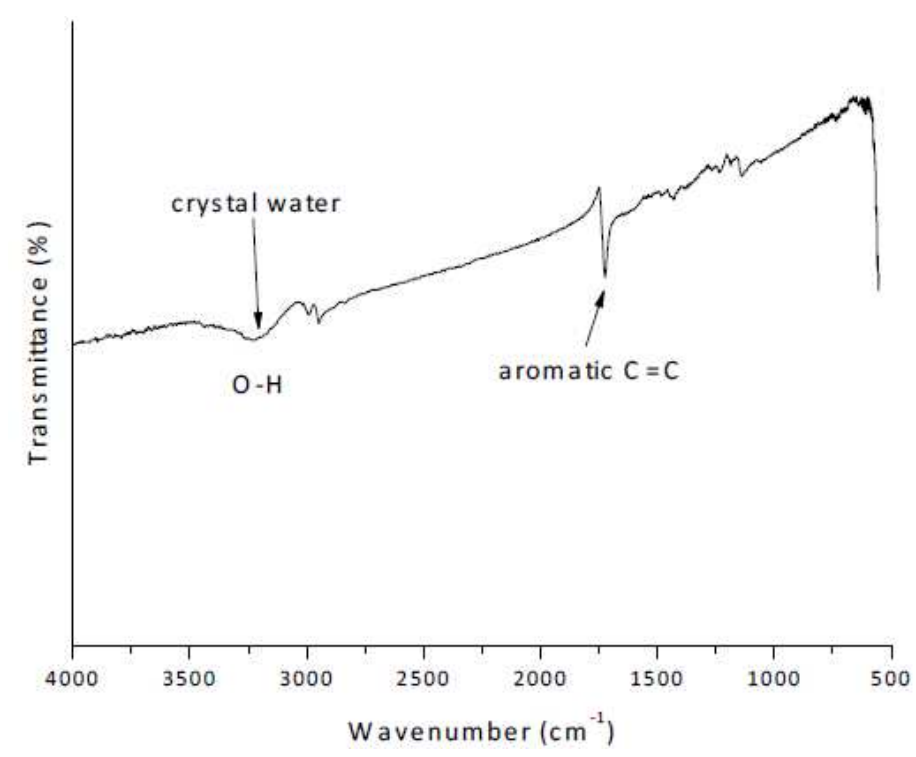

Fig. 11. FTIR spectrum of as-synthesized graphene

Table 1. Summary of the Raman results shown in Fig. 7

\begin{tabular}{lrrrr}
\hline & 1 & 2 & 3 & 4 \\
\hline $\mathrm{D}_{\text {Raman shirf }\left(\mathrm{cm}^{-1}\right)}$ & 1366.90 & 1362.10 & 1364.20 & 1364.20 \\
$\mathrm{G}_{\text {Raman shiff(}\left(\mathrm{cm}^{-1}\right)}$ & 1599.80 & 1599.60 & 1603.20 & 1596.50 \\
$\mathrm{G}^{\prime}{ }_{\text {Raman shirf }\left(\mathrm{cm}^{-1}\right)}$ & 2711.80 & 2708.80 & 2705.50 & 2714.40 \\
$\mathrm{G} / \mathrm{G}^{\prime}{ }_{\text {intensity }}$ & 0.59 & 0.66 & 0.01 & 2.29 \\
$\mathrm{D} / \mathrm{G}_{\text {intensity }}$ & 0.51 & 0.68 & 0.51 & 0.42 \\
\hline
\end{tabular}

By using the approximation of the height profile (L) by the sine function (Troppenz et al., 2013):

$$
L=\int_{0}^{P} \sqrt{1+A^{2} \cos ^{2}\left(\frac{2 \pi x}{P}\right)\left(\frac{2 \pi}{P}\right)^{2}} d x
$$

where, $P$ is the distance between two ripples $(100 \mathrm{~nm})$ and $A$ is their amplitude $(10 \mathrm{~nm})$, we can determine that in our case, an increase of the surface area (ca. 1.23\%) was produced. Hence, the growth of graphene causes a pronounced restructuring of the $\mathrm{Cu}$ surface.

The frictional characteristics of the graphene and $\mathrm{Cu}$ substrate were analyzed by AFM, recording lateral images in contact mode, at different constant loads. The friction force was taken as the average of the half of the differences of the lateral force signal between the back and forth images. The analysis of the friction behavior at the nanoscale, (Friction Force Vs Normal Force) we observe different slopes, related with the friction coefficient, in copper and graphene (Fig. 10). The graphene inherently presents lower resistance to the scanning AFM tip than the metal copper surface, being these values in agreement with the expected for these materials.

Figure 11 shows the FT-IR spectrum of the assynthesized graphene on copper. The measurements were taken in specular reflectance mode. A Kramers Kronig transform was applied to them to get the data in absorbance mode. From this figure, we can observe a strong peak associated with the aromatic $\mathrm{C}=\mathrm{C}(1725$ $\left.\mathrm{cm}^{-1}\right)$, as well as a weak peak at ca. $1430 \mathrm{~cm}^{-1}$, characteristics of reduced graphene. Also the wide band observed at $3223 \mathrm{~cm}^{-1}$ is attributable to crystal water (Abdelsayed et al., 2010; Yuan-Xiang et al., 2014) from the transfer process with PMMA. The absence of peaks from oxygenic functional groups confirms the previously observed by other techniques, that is, graphene samples are not oxidized.

\section{Conclusion}

A novel CVD method, based on plasma-assisted CVD growth, to obtain graphene flakes on copper substrates has been successfully accomplished. The pressure of the reaction mixture (ethanol- $\mathrm{N}_{2}$ ), in presence of a reductive atmosphere at high temperature produces a plasma gas and, as a result, the graphene growth.

To characterize the synthesized graphene, samples were transferred to different substrates by the PMMA process. SEM and HRTEM images confirmed its ordered and crystalline structure. Raman, XPS and FTIR 
techniques were used to characterize the nonoxidized and defect-free nature of the graphene synthesized by this procedure. Finally, by AFM we obtained the graphene and copper substrate morphologies and frictional behaviors. Improving the transfer process of a continuous graphene sheet to other supports, even without PMMA, will be the next step in our research.

\section{Acknowledgement}

The authors thank to "Servicio Interdepartamental de Investigación (SIdI)" at Universidad Autónoma de Madrid for the use of the FESEM and FT-IR facilities. The "Centro Nacional de Microscopía Electrónica" at Universidad Complutense de Madrid is also acknowledged for the use of the TEM facilities. Authors thank to A. del Campo (ICV-CSIC) for his technical assistance in the Raman measurements.

\section{Funding Information}

This work was supported by the Spanish Education and Research Ministry (MEC) under Grant MAT201019804 and from Ministerio de Economía $y$ Competitividad (MINECO) of Spain, through the grant ENE2014-57977-C2-1-R. Financial support from the U.S. Department of Energy, through the Massie Chair Project at Turabo University and from the U.S. Department of Defense under Grant W911NF-14-10046, are also acknowledged.

\section{Author's Contributions}

All authors participated in all experiments, coordinated the data-analysis and contributed to the writing of the manuscript.

\section{Ethics}

This article is original and contains unpublished material. The corresponding author confirms that all of the other authors have read and approved the manuscript and no ethical issues involved.

\section{References}

Abdelsayed, V., S. Moussa, H.M. Hassan, H.S. Aluri and M.M. Collinson et al., 2010. Photothermal deoxygenation of graphite oxide with laser excitation in solution and graphene-aided increase in water temperature. J. Phys. Chem. Lett., 1: 2804-2809. DOI: $10.1021 /$ jz1011143

Batzill, M., 2012. The surface science of graphene: Metal interfaces, CVD synthesis, nanoribbons, chemical modifications and defects. Surface Sci. Rep., 67: 83-115. DOI: 10.1016/j.surfrep.2011.12.001
Campos-Delgado, J., A.R. Botello-Méndez, G. Algara-Siller, B. Hackens and T. Pardoen et al., 2013. CVD synthesis of mono- and few-layer graphene using alcohols at low hydrogen concentration and atmospheric pressure. Chem. Phys. Lett., 584: 142-146.

DOI: 10.1016/j.cplett.2013.08.031

Casero, E., C. Alonso, L. Vázquez, M.D. Petit-Domínguez and A.M. Parra-Alfambra et al., 2013. Comparative response of biosensing platforms based on synthesized graphene oxide and electrochemically reduced graphene. Electroanalysis, 25: 154-165.

DOI: 10.1002/elan.201200480

Dacheng, W., W. Bin, G. Yunlong, G. Yu and Y. Liu et al., 2013. Controllable chemical vapor deposition growth of few layer graphene for electronic devices. Accounts Chem. Res., 46: 106-115. DOI: $10.1021 / \operatorname{ar} 300103 \mathrm{f}$

Dacheng, W. and L. Yunqi, 2010. Controllable synthesis of graphene and its applications. Adv. Mater., 22: 3225-3241. DOI: 10.1002/adma.200904144

Dresselhaus, M.S., A. Jorio, M. Hofmann, G. Dresselhaus and R. Saito et al., 2010. Perspectives on carbon nanotubes and graphene raman spectroscopy. Nano Lett., 10: 751-758. DOI: 10.1021/n1904286r

Edwards, R.S. and K.S. Coleman, 2013. Graphene film growth on polycrystalline metals. Accounts Chem. Res., 46: 23-30. DOI: 10.1021/ar3001266

Ferrari, A.C., 2007. Raman spectroscopy of graphene and graphite: Disorder, electron-phonon coupling, doping and nonadiabatic effects. Solid State Commun., 143: 47-57. DOI: $10.1016 /$ j.ssc.2007.03.052

Fisichella, G., S. Di Franco, P. Fiorenza, R.L. Nigro and F. Roccaforte et al., 2013. Micro and nanoscale electrical characterization of large-area graphene transferred to functional substrates. Beilstein J. Nanotechnol., 4: 234-242. DOI: 10.3762/bjnano.4.24

Gao, L., J.R. Guest and N.P. Guisinger, 2010. Epitaxial graphene on $\mathrm{Cu}(111)$. Nano Lett., 10: 3512- 3516. DOI: $10.1021 / \mathrm{nl} 1016706$

Goldoni, A., R. Larciprete, L. Gregoratti, B. Kaulich and M. Kiskinova et al., 2002. X-ray photoelectron microscopy of the $\mathrm{C} \square$ 1s core level of free-standing single-wall carbon nanotube bundles. Applied Phys. Lett., 80: 2165-2167. DOI: 10.1063/1.1464217

Guermoune, A., T. Chari, F. Popescu, S.S. Sabri and J. Guillemette et al., 2011. Chemical vapor deposition synthesis of graphene on copper with methanol, ethanol and propanol precursors. Carbon, 49: 4204-4210. DOI: 10.1016/j.carbon.2011.05.054

Guoxiu, W., J. Yang, J. Park, X. Gou and B. Wang et al., 2008. Facile synthesis and characterization of graphene nanosheets. J. Phys. Chem. C, 112: 8192-8195. DOI: $10.1021 /$ jp710931h 
Kudin, K.N., B. Ozbas, H.C. Schniepp, R.K. Prud'homme and I.A. Aksay et al., 2008. Raman spectra of graphite oxide and functionalized graphene sheets. Nano Lett., 8: 36-41. DOI: 10.1021/nl071822y

Malard, L.M., M.A. Pimenta, G. Dresselhaus and M.S. Dresselhaus, 2009. Raman spectroscopy in graphene. Phys. Rep., 473: 51-87. DOI: 10.1016/j.physrep.2009.02.003

Meyer, J.C., A.K. Geim, M.I. Katsnelson, K.S. Novoselov and T.J. Booth et al., 2007. The structure of suspended graphene sheets. Nature, 446: 60-63. DOI: 10.1038 /nature 05545

Morant, C., T. Campo, F. Márquez, C. Domingo and J.M. Sanz et al., 2012. Mo-Co catalyst nanoparticles: Comparative study between TiN and $\mathrm{Si}$ surfaces for single-walled carbon nanotube growth. Thin Solid Films, 520: 5232-5238.

DOI: $10.1016 /$ j.tsf.2012.03.099

Phaedon, A., 2010. Graphene: Electronic and photonic properties and devices. Nano Lett., 10: 4285-4294. DOI: $10.1021 / \mathrm{nl} 102824 \mathrm{~h}$

Schwierz, F., 2010. Graphene transistors. Nature Nanotechnol., 5: 487-496. DOI: $10.1038 /$ nnano.2010.89

Songfeng, P., Z. Jinping, D. Jinhong, W. Ren and H.M. Cheng, 2010. Direct reduction of graphene oxide films into highly conductive and flexible graphene films by hydrohalic acids. Carbon, 48: 4466-4474. DOI: 10.1016/j.carbon.2010.08.006
Troppenz, G.V., M.A. Gluba, M. Kraft, J. Rappich and N.H. Nickel, 2013. Strain relaxation in graphene grown by chemical vapor deposition. J. Applied Phys., 114: 214312. DOI: 10.1063/1.4834538

Varela-Rizo, H., I. Rodriguez-Pastor, C. Merino and I. Martin-Gullon, 2010. Highly crystalline graphene oxide nano-platelets produced from helical-ribbon carbon nanofibers. Carbon, 48: 3640-3643. DOI: $10.1016 /$ j.carbon.2010.05.033

Xuesong, L., C. Weiwei, A. Jinho, S. Kim and J. Nah et al., 2009. Large-area synthesis of high-quality and uniform graphene films on copper foils. Science, 324: 1312-1314. DOI: 10.1126/science.1171245

Yuan-Xiang, F., W. Xiao-Ming, M. Dong-Chuan and S.S. Lu, 2014. Production of monolayer, trilayer and multi-layer graphene sheets by a re-expansion and exfoliation method. J. Mater. Sci., 49: 2315-2323. DOI: $10.1007 / \mathrm{s} 10853-013-7930-4$

Guo, Y., B. Wu, H. Liu, Y. Ma and Y. Yang et al., 2011. Electrical assembly and reduction of graphene oxide in a single solution step for use in flexible sensors. Adv. Mater., 23: 4626-4630. DOI: 10.1002/adma.201103120

Zhang, Y., L. Zhang and C. Zhou, 2013. Review of chemical vapor deposition of graphene and related applications. Accounts Chem. Res., 10: 2329-2339. DOI: $10.1021 / \operatorname{ar} 300203 n$ 